Advances in Solid State Physics Volume 48 


\section{Advances in Solid State Physics}

Advances in Solid State Physics is a book series with a history of about 50 years. It contains the invited lectures presented at the Spring Meetings of the "Arbeitskreis Festkörperphysik" of the "Deutsche Physikalische Gesellschaft", held in March of each year. The invited talks are intended to reflect the most recent achievements of researchers working in the field both in Germany and worldwide. Thus the volume of the series represents a continuous documentation of most recent developments in what can be considered as one of the most important and active fields of modern physics. Since the majority of invited talks are usually given by young researchers at the start of their career, the articles can also be considered as indicating important future developments.

The speakers of the invited lectures and of the symposia are asked to contribute to the yearly volumes with the written version of their lecture at the forthcoming Spring Meeting of the Deutsche Physikalische Gesellschaft by the Series Editor. Colored figures are available in the online version for some of the articles.

Advances in Solid State Physics is addressed to all scientists at universities and in industry who wish to obtain an overview and to keep informed on the latest developments in solid state physics. The language of publication is English.

\section{Series Editor}

Prof. Dr. Rolf Haug

Abteilung Nanostrukturen

Institut für Festkörperphysik

Universität Hannover

Appelstr. 2

30167 Hannover

Germany

haug@nano.uni-hannover.de 


\author{
Rolf Haug (Ed.)
}

\title{
Advances in Solid State Physics 48
}

With 182 Figures and 13 Tables

Springer 
Prof. Dr. Rolf Haug

Abteilung Nanostrukturen

Institut für Festkörperphysik

Universität Hannover

Appelstr. 2

30167 Hannover

Germany

haug@nano.uni-hannover.de

ISSN print edition: $1438-4329$

ISSN electronic edition: 1617-5034

ISBN: 978-3-540-85858-4

e-ISBN: 978-3-540-85859-1

DOI 10.1007/978-3-540-85859-1

Physics and Astronomy Classification Scheme (PACS): 60.00; 70.00; 80.00

Library of Congress Control Number: 2008935399

(c) Springer-Verlag Berlin Heidelberg 2009

This work is subject to copyright. All rights are reserved, whether the whole or part of the material is concerned, specifically the rights of translation, reprinting, reuse of illustrations, recitation, broadcasting, reproduction on microfilm or in any other way, and storage in data banks. Duplication of this publication or parts thereof is permitted only under the provisions of the German Copyright Law of September 9, 1965 , in its current version, and permission for use must always be obtained from Springer. Violations are liable to prosecution under the German Copyright Law.

The use of general descriptive names, registered names, trademarks, etc. in this publication does not imply, even in the absence of a specific statement, that such names are exempt from the relevant protective laws and regulations and therefore free for general use.

Cover concept using a background picture by Dr. Ralf Stannarius, Faculty of Physics and Earth Sciences, Institute of Experimental Physics I, University of Leipzig, Germany

Cover design: WMXDesign GmbH, Heidelberg

Printed on acid-free paper

$\begin{array}{lllllllll}9 & 8 & 7 & 6 & 5 & 4 & 3 & 2 & 1\end{array}$

springer.com 


\section{Preface}

The 2008 spring meeting of the Arbeitskreis Festkörperphysik was held in Berlin between February 24 and 29, 2008 in conjunction with the 72. Annual Meeting of the Deutsche Physikalische Gesellschaft. It was the last spring meeting of the Arbeitskreis Festkörperphysik because in the meantime it changed its name to Sektion kondensierte Materie which better shows that it also includes soft materials and biological systems. Therefore, the next spring meeting in Dresden will be organized by the Sektion kondensierte Materie of the Deutsche Physikalische Gesellschaft. The number of participants of this year's meeting in Berlin exceeded 5600 and there were more than 4600 scientific contributions. With these numbers this meeting was the largest physics meeting in Europe and among the largest physics meetings in the world in 2008.

The present volume, 48 of the Advances in Solid State Physics contains the written version of a large number of the invited talks in Berlin and gives a nice overview of the present status of condensed matter physics. Low-dimensional systems are dominating the field and especially nanowires and quantum dots. In recent years one learned how to produce nanowires directly during a growth process. Therefore, a number of articles is related to such nanowires. In nanoparticles and quantum dots the dimensionality is further reduced and we learn more and more how to produce such systems and what effects result from the confinement in all three dimensions. Spin effects and magnetism is another important field of present day research in solid state physics. The third chapter covers this physics including an article about graphene. The growing interest into organic materials and biological systems is reflected in a large chapter of this book with the title Organic Materials and Water. The last chapters of this book cover aspects which range from dynamical effects to device physics and characterization tools. 


\section{Contents}

\section{Part I Nanowires}

From Ordered Arrays of Nanowires to Controlled Solid State Reactions

Margit Zacharias and Hong Jin Fan......................... 3

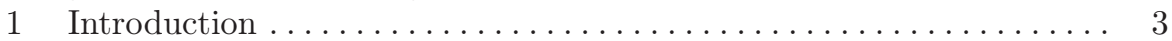

2 Methods for nano-patterning ....................... 6

3 Selected results - stimulated emission $\ldots \ldots \ldots \ldots \ldots \ldots \ldots .7$

4 Nanotubes based on Kirkendall diffusion and solid state reactions . . 8

$5 \quad$ Summary .................................. 11

6 Acknowledgement ............................... 12

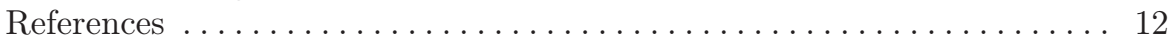

Growth Methods and Properties of High Purity III-V

Nanowires by Molecular Beam Epitaxy

D. Spirkoska, C. Colombo, M. Heiß, M. Heigoldt, G. Abstreiter, and

A. Fontcuberta i Morral ................................ 13

1 Introduction ................................... 13

2 Experimental .................................. 14

3 Selective area epitaxy ............................ 14

4 Conditions leading to group III assisted growth of nanowires ...... 17

5 Structural and optical properties . ..................... 19

6 Potential for future structures and devices ................ 21

7 Conclusions.................................. 23

8 Acknowledgements .............................. 23

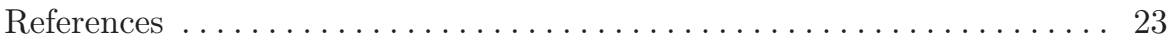

Simple Ways to Complex Nanowires and Their Application Mady Elbahri, Seid Jebril, Sebastian Wille, and Rainer Adelung....... 27

1 Introduction ................................. 27

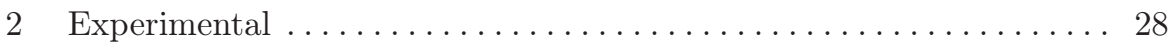




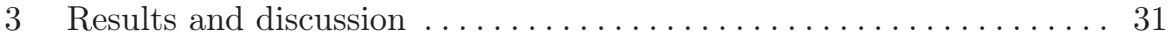

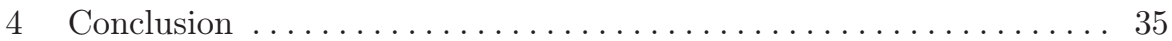

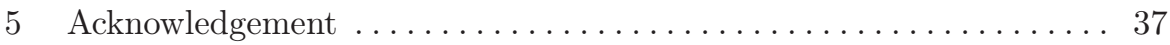

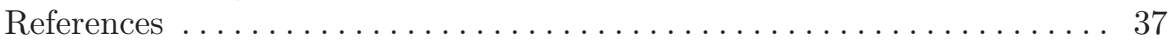

ZnO Nanostructures: Optical Resonators and Lasing

Klaus Thonke, Anton Reiser, Martin Schirra, Martin Feneberg,

Günther M. Prinz, Tobias Röder, Rolf Sauer, Johannes Fallert,

Felix Stelzl, Heinz Kalt, Stefan Gsell, Matthias Schreck, and

Bernd Stritzker .................................. 39

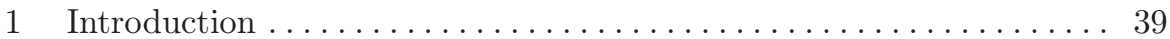

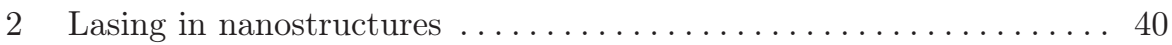

3 Single $\mathrm{ZnO}$ pillars as nano-resonators $\ldots \ldots \ldots \ldots \ldots \ldots \ldots \ldots \ldots 4$

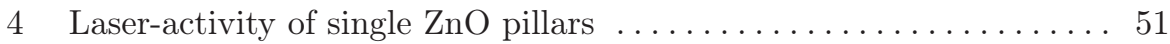

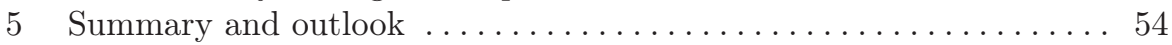

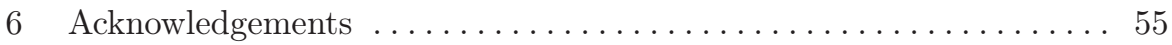

References ................................. 55

\section{Waveguiding and Optical Coupling in $\mathrm{ZnO}$ Nanowires and Tapered Silica Fibers}

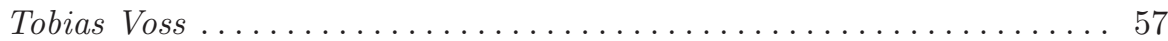

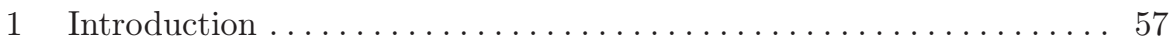

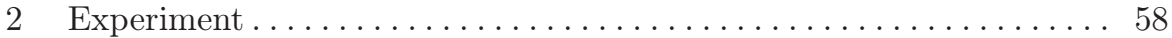

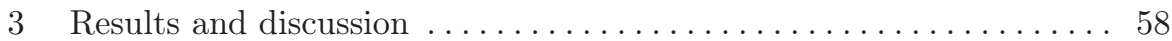

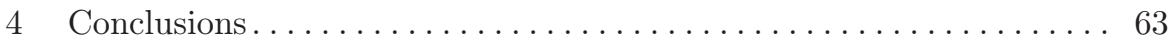

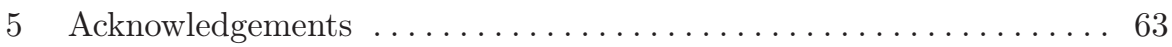

References ............................... 64

\section{Part II Quantum Dots and Nanoparticles}

\section{Electrically Driven Single Quantum Dot Emitter Operating} at Room Temperature

Tilmar Kümmell, Robert Arians, Arne Gust, Carsten Kruse, Sergey

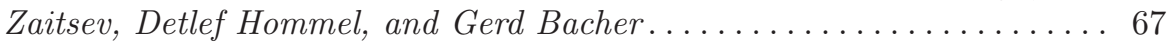

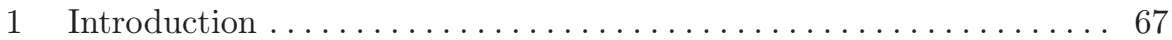

2 Quantum dots optimized for RT emission................. 68

3 Electrically driven single quantum dot emitter $\ldots \ldots \ldots \ldots \ldots \ldots 73$

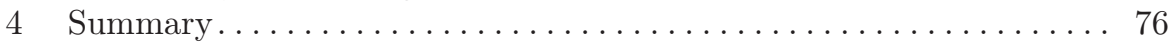

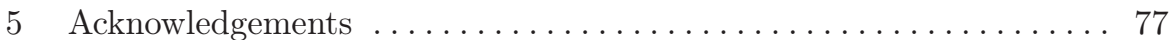

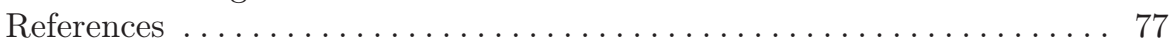


Silicon Nanoparticles: Excitonic Fine Structure and Oscillator Strength

Cedrik Meier, Stephan Lüttjohann, Matthias Offer, Hartmut Wiggers, and Axel Lorke . . . . . . . . . . . . . . . . . . . . . . . . . . . . . 79

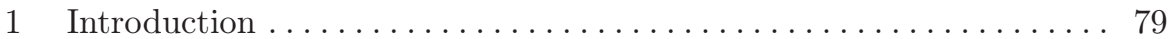

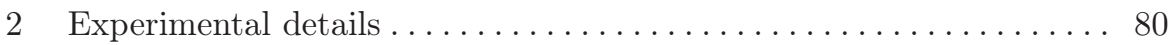

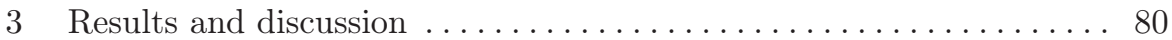

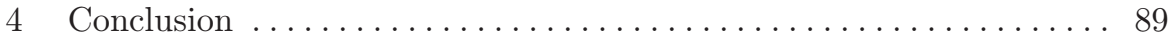

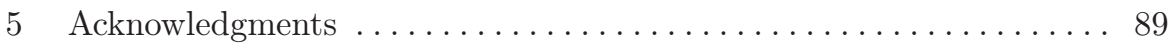

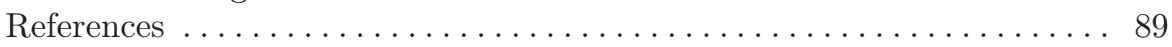

Intrinsic Non-Exponential Decay of Time-Resolved Photoluminescence from Semiconductor Quantum Dots

Jan Wiersig, Christopher Gies, Norman Baer, and Frank Jahnke . . . . . 91

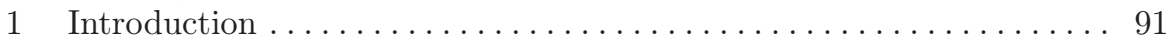

2 Time-resolved photoluminescence ................... 92

3 Correlations . . . . . . . . . . . . . . . . . . . . . . . . . . . . 95

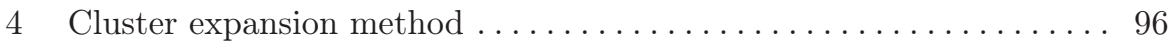

5 Numerical results: non-exponential PL decay . . . . . . . . . . . . 97

6 Numerical results: excitation-intensity dependence . . . . . . . . . . . 99

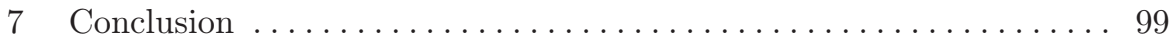

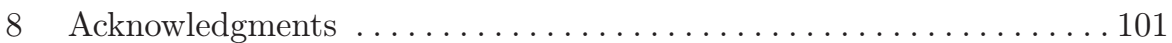

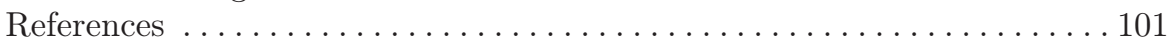

Electrical Spin Injection into Single InGaAs Quantum Dots

Michael Hetterich, Wolfgang Löffler, Pablo Aßhoff, Thorsten Passow, Dimitri Litvinov, Dagmar Gerthsen, and Heinz Kalt. . . . . . . . . . . . 103

1 Introduction . . . . . . . . . . . . . . . . . . . . . . . . . 103

2 Initialization and readout of spins in single dots . . . . . . . . . . 105

3 Spin loss mechanisms and device optimization . . . . . . . . . . . 107

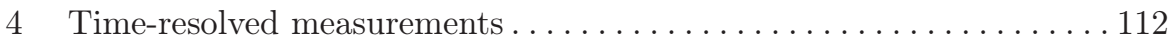

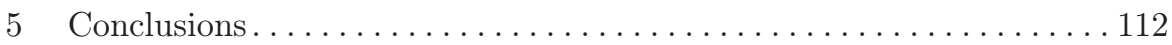

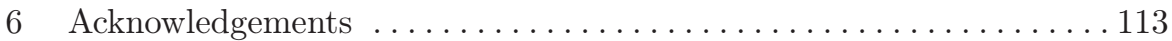

References .................................... 113

\section{Part III Spin and Magnetism}

\section{Spintronic and Electro-Mechanical Effects in Single-Molecule} Transistors

Maarten R. Wegewijs, Felix Reckermann, Martin Leijnse, and Herbert

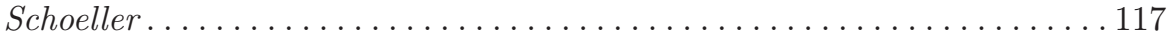

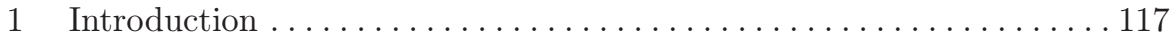

2 Mixed-valence dimer transistor . . . . . . . . . . . . . . . . . . . . . . . . . . 119

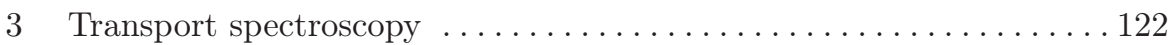

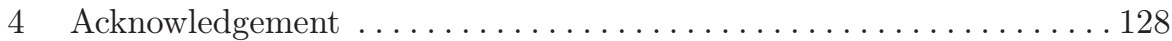

References .................................... 128 
Transport in 2DEGs and Graphene: Electron Spin vs. Sublattice Spin

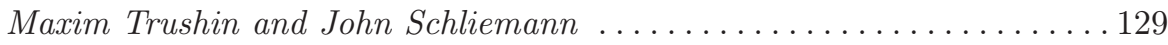

1 Introduction . . . . . . . . . . . . . . . . . . . . . . . . . . . . 129

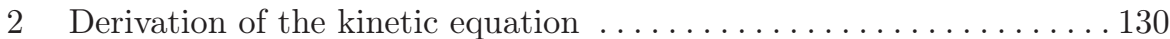

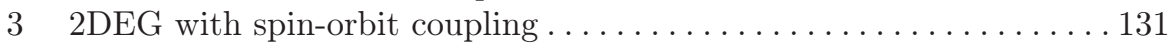

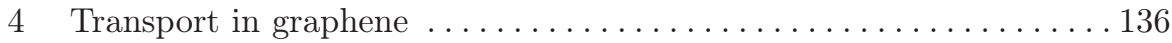

5 Conculsion remarks . . . . . . . . . . . . . . . . . . . . . . 139

References .................................... 140

\section{Spin Dynamics in High-Mobility Two-Dimensional Electron} Systems

Tobias Korn, Dominik Stich, Robert Schulz, Dieter Schuh, Werner

Wegscheider, and Christian Schüller.................... 143

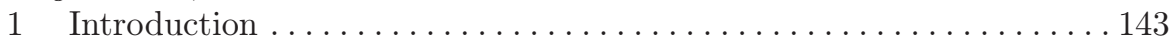

2 Theory................................. 144

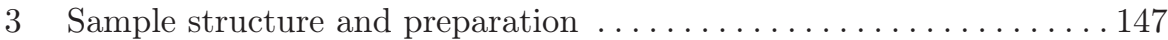

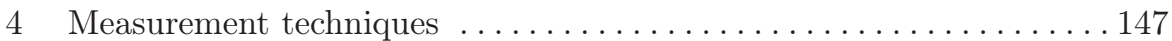

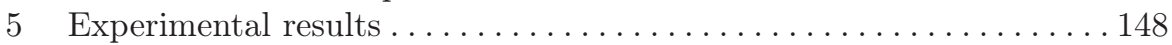

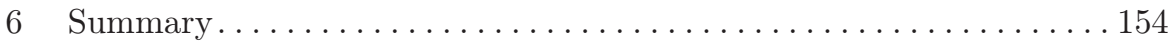

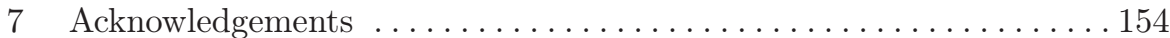

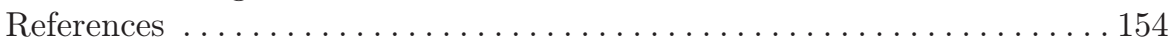

Magnetization Dynamics of Coupled FerromagneticAntiferromagnetic Thin Films

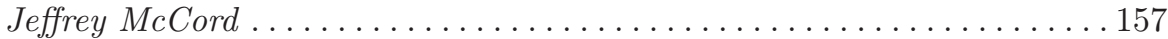

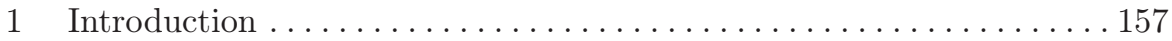

2 Magnetization dynamics. . . . . . . . . . . . . . . . . . 158

3 Statics and dynamics of exchange biased $\mathrm{F} / \mathrm{AF}$ films . . . . . . . . . 160

$4 \mathrm{~F} / \mathrm{AF} / \mathrm{F}$ structures below the onset of exchange bias . . . . . . . . . 163

5 Conclusions..................................... 168

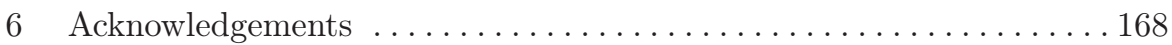

References ...................................... 168

\section{Magnetic and Electronic Properties of Heusler Alloy Films}

Investigated by X-Ray Magnetic Circular Dichroism

Hans-Joachim Elmers, Andres Conca, Tobias Eichhorn, Andrei

Gloskovskii, Kerstin Hild, Gerhard Jakob, Martin Jourdan, and

Michael Kallmayer.................................171

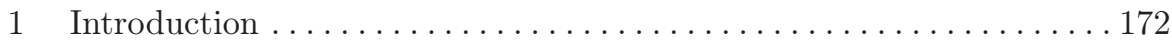

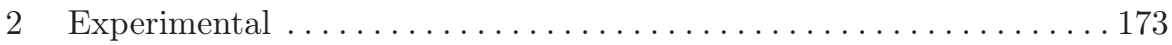

3 Martensitic phase transition in $\mathrm{Ni}_{2} \mathrm{MnGa}$ films . . . . . . . . . . . . 173

4 Interface properties of Heusler compound films . . . . . . . . . . . . . . . . 177

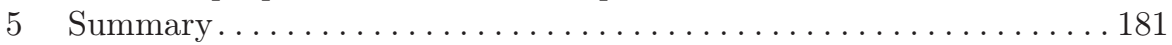

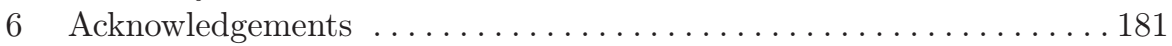

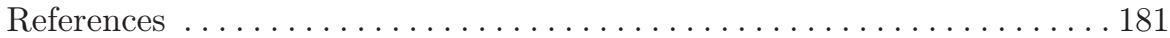


Coherent Spin Dynamics in Nanostructured SemiconductorFerromagnet Hybrids

Patric Hohage, Jörg Nannen, Simon Halm, and Gerd Bacher ......... 183

1 Introduction . . . . . . . . . . . . . . . . . . . . . 183

2 Samples and experiment .......................... 184

3 Free vs. localized spin precession in a semiconductor . . . . . . . . . 185

4 Spin dynamics in ferromagnet-semiconductor hybrids . . . . . . . . . 187

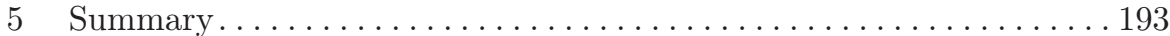

6 Acknowledgements . . . . . . . . . . . . . . . . . . . . . 194

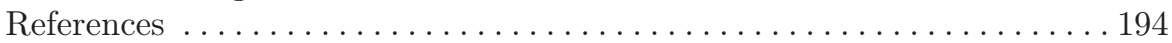

\section{Part IV Organic Materials and Water}

\section{Coupling of Paramagnetic Biomolecules to Ferromagnetic Surfaces}

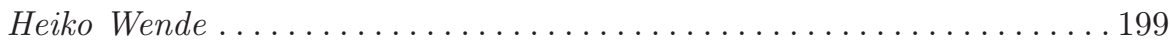

1 Introduction . . . . . . . . . . . . . . . . . . . . . . . . . . . 199

2 Experimental details .............................. 200

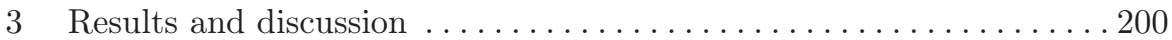

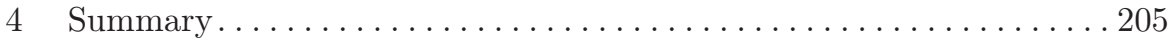

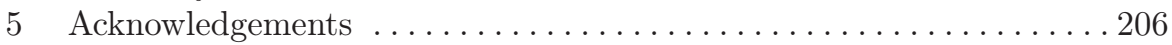

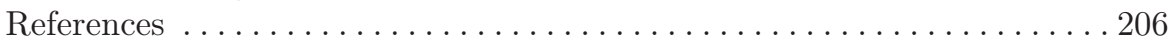

\section{Band Alignment in Organic Materials}

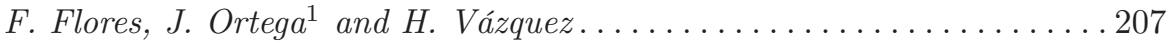

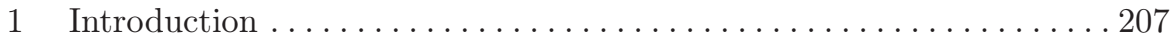

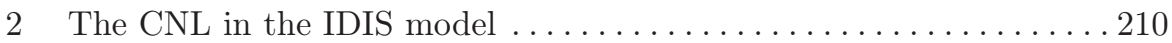

3 The IDIS model at MO interfaces $\ldots \ldots \ldots \ldots \ldots \ldots \ldots \ldots \ldots \ldots \ldots \ldots$

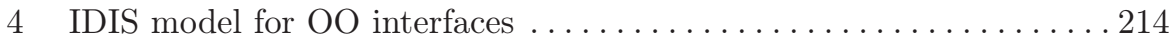

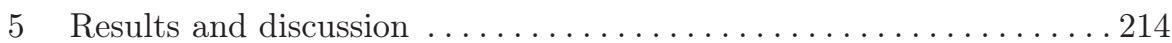

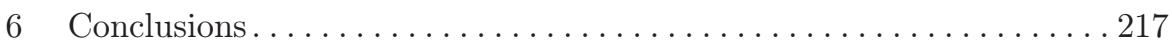

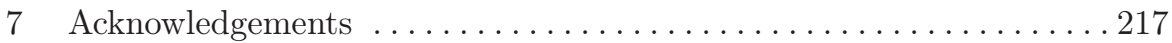

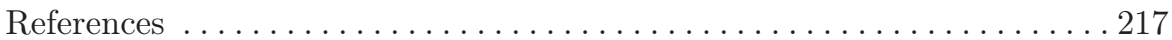

Organometallic Nanojunctions Probed by Different

Chemistries: Thermo-, Photo-, and Mechano-Chemistry

Martin Konôpka, Robert Turanský, Nikos L. Doltsinis, Dominik Marx, and Ivan Śtich . . . . . . . . . . . . . . . . . . . . . . . . . . . . . . . . . . . . . . . . . 219

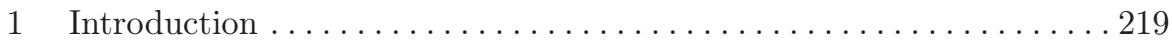

2 Thermo- and Mechano-chemistry of copper-ethylthiolate junctions . . 220

3 Mechanically and opto-mechanically controlled azobenzene (AB) switch based on AB-gold break-junction. . . . . . . . . . . . . . 225

4 Conclusions... . . . . . . . . . . . . . . . . . . . . . . . . . . . . . . 231

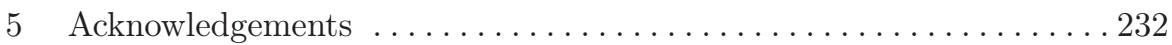

References ........................................ 233 
When It Helps to Be Purely Hamiltonian: Acceleration of Rare Events and Enhanced Escape Dynamics

Dirk Hennig, Simon Fugmann, Lutz Schimansky-Geier, and

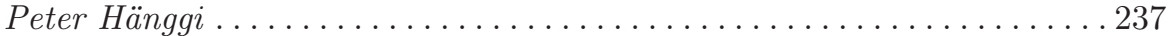

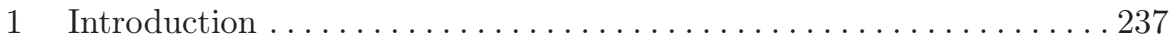

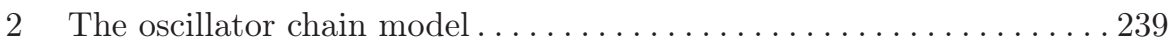

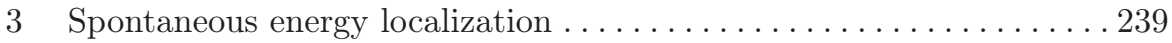

4 Escape dynamics . . . . . . . . . . . . . . . . . . . . . . . . . 242

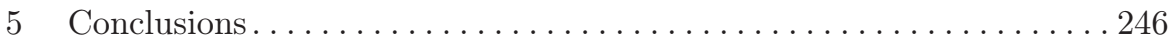

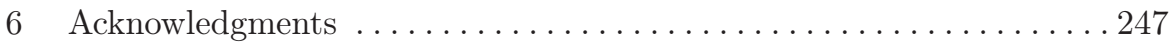

References ................................... 247

Liquid Polyamorphism and the Anomalous Behavior of Water

H. E. Stanley, S. V. Buldyrev, S.-H. Chen, G. Franzese, S. Han,

P. Kumar, F. Mallamace, M. G. Mazza, L. Xu, and Z. Yan ......... 249

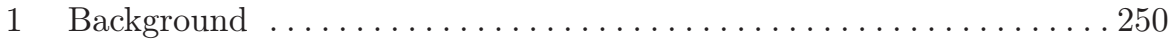

2 Understanding "static heterogeneities" . . . . . . . . . . . . . . . . . . 252

3 Understanding "dynamic heterogeneities" . . . . . . . . . . . . . . . . 257

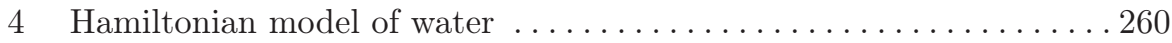

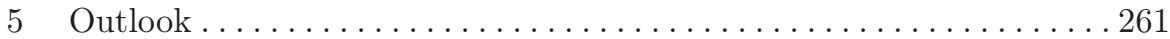

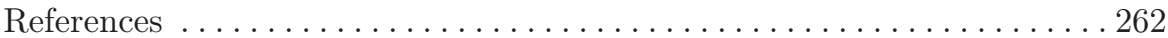

\section{Part V Dynamical Effects, Rectification and Nonlinearities}

Terahertz Detection of Many-Body Signatures in Semiconductor Heterostructures

Sangam Chatterjee, Torben Grunwald, Stephan W. Koch, Galina

Khitrova, Hyatt M. Gibbs, and Rudolf Hey .................. 269

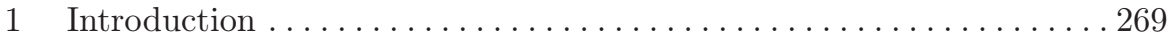

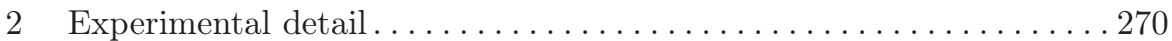

3 Data analysis . . . . . . . . . . . . . . . . . . . 272

4 Intra-excitonic 1s-2p transition in GaAs/(AlGa)As quantum wells . . 274

5 Intra-excitonic 1s-2p transition in (GaIn)As/GaAs quantum wells . . 276

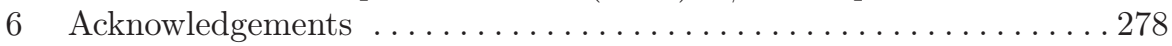

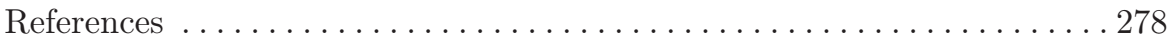

Theory of Ultrafast Dynamics of Electron-Phonon Interactions in Two Dimensional Electron Gases: Semiconductor Quantum Wells, Surfaces and Graphene

Marten Richter, Stefan Butscher, Norbert Bücking, Frank Milde,

Carsten Weber, Peter Kratzer, Matthias Scheffler, and Andreas Knorr . 281

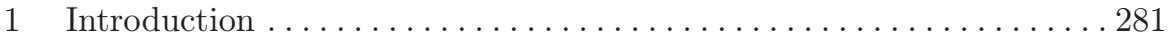

2 Theoretical framework . . . . . . . . . . . . . . . . . . 282

3 Phonon-induced relaxation dynamics at the silicon (001) $2 \times 1$ surface . . . . . . . . . . . . . . . . . . . . . . . . . . . . . . . 284 
4 Scattering response and spatiotemporal wavepackets in quantum cascade lasers . . . . . . . . . . . . . . . . . . . . . . . . 286

5 Non-equilibrium phonon dynamics in graphene . . . . . . . . . . . 287

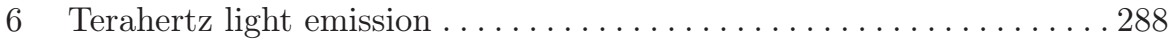

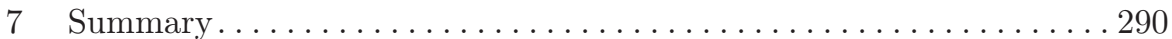

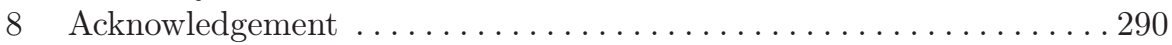

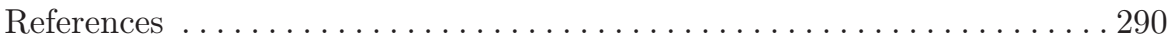

\section{Optical Microcavities as Quantum-Chaotic Model Systems:} Openness Makes the Difference!

Martina Hentschel . . . . . . . . . . . . . . . . . . . . . . . . . . . . . . . . 293

1 Introduction . . . . . . . . . . . . . . . . . . . . . . . . . . . 293

2 Deviations from ray-wave correspondence. . . . . . . . . . . . . . . . 297

3 Correcting ray optics by wave effects: Goos-Hänchen shift and Fresnel filtering . . . . . . . . . . . . . . . . . . 300

4 Outlook: non-Hamiltonian dynamics in quantum-chaotic model

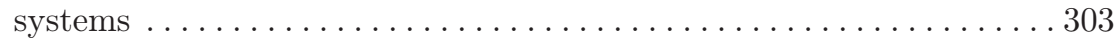

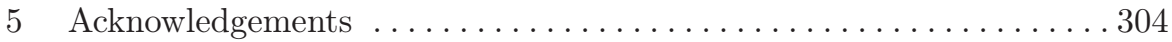

References ...................................... 304

Nonlinear Transport Properties of Electron Y-Branch Switches

Lukas Worschech, David Hartmann, Stefan Lang, D. Spanheimer,

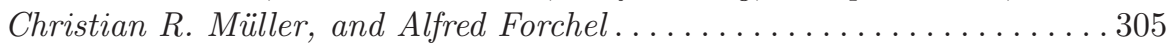

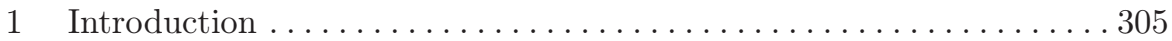

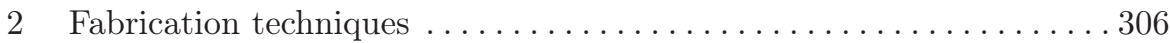

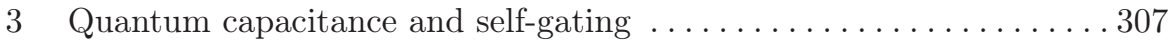

4 Self-gating in a Y-branch switch at room temperature . . . . . . . . . 308

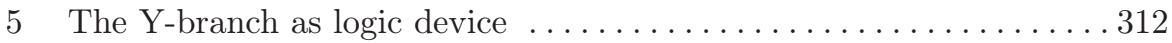

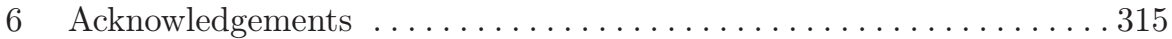

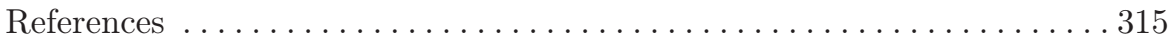

Rectification through Entropic Barriers

Gerhard Schmid, P. Sekhar Burada, Peter Talkner, and Peter Hänggi . . 317

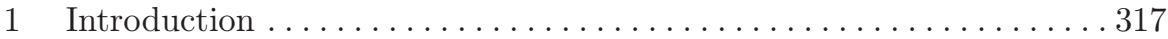

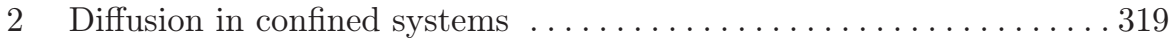

3 Transport in periodic channels with broken symmetry . . . . . . . . . 320

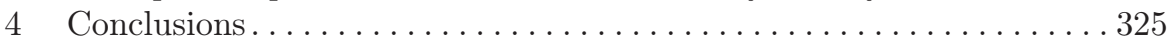

5 Acknowledgements . . . . . . . . . . . . . . . . . . . . . . . . 326

References ................................ 326 


\section{Part VI Characterization of Materials and Devices}

Microstructure Tomography - An Essential Tool to Understand 3D Microstructures and Degradation Effects

Alexandra Velichko and Frank Mücklich . . . . . . . . . . . . . . . . . . . 331

1 Introduction . . . . . . . . . . . . . . . . . . . . . . . . . . . 331

2 Basic characteristics of the microstructure . . . . . . . . . . . . 332

3 Determination of the $3 \mathrm{D}$ grain size distribution from $2 \mathrm{D}$

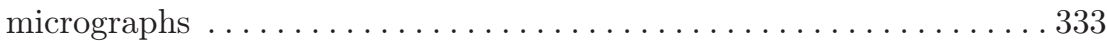

4 Analysis of the 3D tomographical images . . . . . . . . . . . . 335

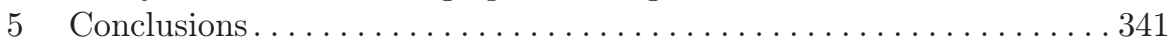

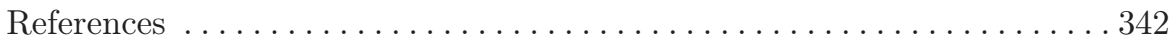

\section{Profiling of Fiber Texture Gradients by Anomalous X-ray Diffraction}

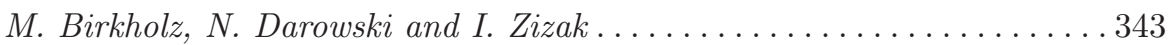

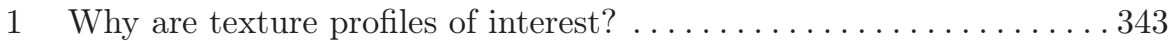

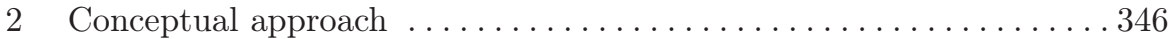

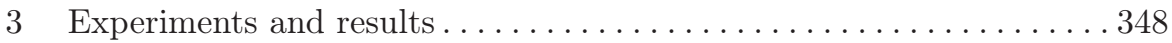

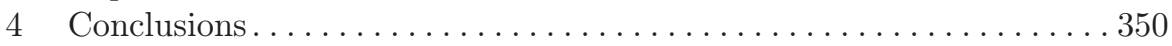

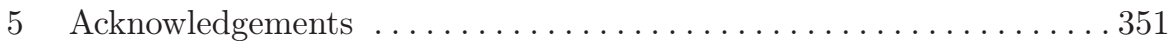

References ...................................... 351

Film Production Methods in Precision Optics

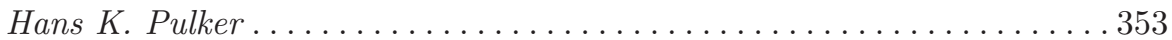

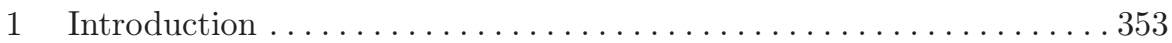

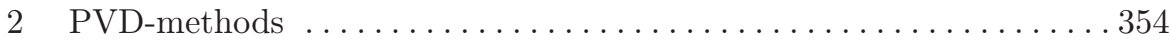

3 Thin film properties ............................ 364

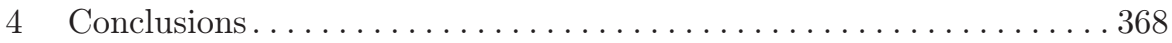

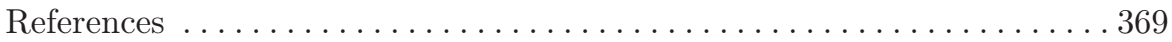

Advanced Metrology for Next Generation Transistors

Alain C. Diebold .................................. 371

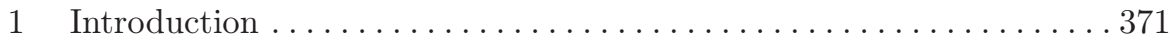

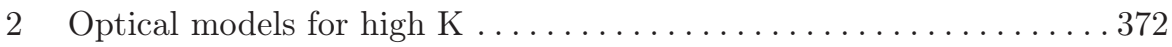

3 Optical model for metal films . . . . . . . . . . . . . . . . . . 377

4 Charge pumping based capacitance - voltage measurements . . . . . . 377

5 Optical measurement of charge trapped in high $\mathrm{k}$ and interface . . . . 378

6 Measurement of ultra-thin SOI and observation of quantum confinement . . . . . . . . . . . . . . . . . . . . . . . . . . . 380

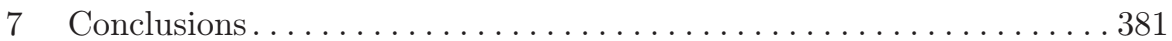

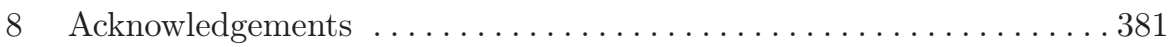

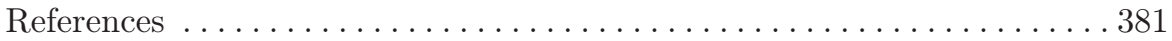

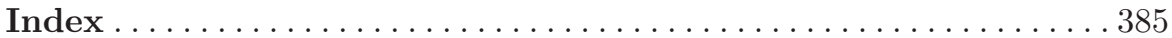

